\title{
Control of Low-Frequency Oscillation in a Hall Thruster
}

\author{
By Takeshi Furukawa, ${ }^{1)}$ Takeshi MiYasakA ${ }^{2)}$ and Toshi FujiwarA ${ }^{2)}$ \\ ${ }^{1)}$ Graduate School, Nagoya University, Nagoya, Japan \\ ${ }^{2)}$ Department of Aerospace Engineering, Nagoya University, Nagoya, Japan
}

(Received February 27th, 2001)

\begin{abstract}
The studies of low-frequency discharge current oscillation phenomena in the $20 \mathrm{kHz}$ range causing a deterioration of propulsive efficiency and operational instability are important to improve Hall thruster performance. A possibility that the amplitude of $20 \mathrm{kHz}-$ range oscillation could be controlled by changing ionization-zone length was predicted in our previous work, based on experiments and theoretical analyses. ${ }^{1)}$ In this paper, the physical mechanisms of the ionization process in the acceleration channel are studied by using an unsteady one-dimensional numerical analysis; the methods of controlling oscillation amplitude are concretely discussed from the viewpoint of ionization-zone length. The numerical code generated in the present study would be an effective tool for the best design of a Hall thruster.
\end{abstract}

Key Words: Electric Propulsion, Hall Thruster, Numerical Analysis, Discharge Current Oscillation

\section{Nomenclature}

(SI units unless noted otherwise)

\begin{aligned}$B: &$ magnetic field \\ $D_{\mathrm{e}}: &$ diffusion coefficient \\ $D_{\mathrm{e}}^{\mathrm{T}}: &$ thermal-diffusion coefficient \\ $E: &$ electric field \\ $e: &$ electric discharge \\ $I_{\mathrm{T}}: &$ total current \\ $k: &$ Boltzmann constant \\ $L_{\mathrm{c}}: &$ acceleration channel length \\ $L_{\mathrm{i}, \mathrm{eq}}: &$ equilibrium length of ionization zone \\ $L_{\mathrm{i}}: &$ ionization-zone length \\ $m: &$ particle mass \\ $\dot{m}: &$ mass flow rate of propellant (Xe) \\ $n: &$ number density \\ $r_{1}: &$ inner radius of acceleration channel \\ $r_{2}: &$ outer radius of acceleration channel \\ $S: &$ cross-section of channel \\ $T: &$ temperature \\ $t: &$ time \\ $V_{\mathrm{exit}}: &$ voltage at channel exit \\ $V_{\mathrm{d}}: &$ discharge voltage \\ $V_{\mathrm{i}}: &$ ionization voltage \\ $v: &$ velocity \\ $z: &$ distance from anode \\ $\beta: &$ excitation parameter \\ $\Gamma: &$ flux \\ $\delta: &$ secondary-electron emission coefficient \\ $\varepsilon_{\mathrm{i}}: &$ ionization energy \\ $v_{\mathrm{L}}: &$ ion-loss rate per unit volume \\ $v_{\mathrm{P}}: &$ ion-production rate per unit volume \\ $\sigma: &$ ionization cross-section \\ & \\ \hline & \end{aligned}

(C) 2001 The Japan Society for Aeronautical and Space Sciences

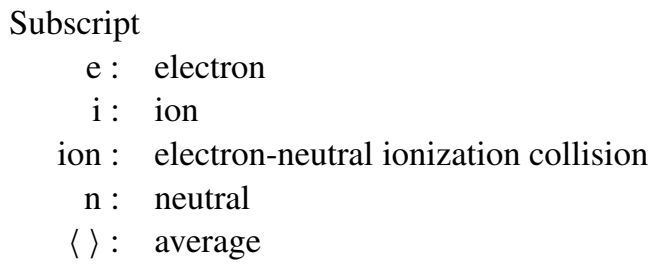

\section{Introduction}

\subsection{Physics on hall thruster}

The Hall thruster is a promising propulsion device for space missions capable of reducing mass flow rate of a propellant resulting from high specific impulse (1,000-3,000 s). It has been used more than a hundred times chiefly as a thruster for satellite station keeping and orbit correction. ${ }^{2}$ Moreover, it has the possibility of achieving thrust density identical to the electromagnetic-acceleration-type thrusters under no restriction of the space charge limited current rule; the acceleration part is quasi-neutral. ${ }^{3)}$ High-powered Hall thrusters of the $50-100 \mathrm{~kW}$ class have been developed to acquire high performance (SPT290, for example, giving about $1,500 \mathrm{mN}$ ) and are expected to shorten the flight time in Mars missions and others. ${ }^{4)}$

The development of the Hall thruster originated in Russia where their research results overwhelmed other countries. Many Hall thrusters manufactured there are called Stationary Plasma Thrusters (SPTs) that are characterized in having a comparatively long acceleration channel and a distribution of magnetic field lines concentrated around the channel exit. Recently, studies of SPT on the reevaluation of performance for verifying the possibility of practical use and on the measurement of beam characteristics and thruster abrasion during continuous operation have been actively performed in Europe and the United States. Also, when the thruster is loaded on a satellite, serious problems of insulator wall 
abrasion and a deposition of thruster material over the main body of the satellite could develop; they have been studied from the viewpoint of plasma in channel and in plume. ${ }^{5)}$ The necessity of physics-related research on plasma characteristics in Hall thrusters has originated from such backgrounds. However, the couplings between electric and magnetic fields and among electron, ion, and neutral transports are complicated in them, where the plasma properties are far from being clearly understood. ${ }^{2)}$

\subsection{Low-frequency oscillation}

Some serious problems still remain for the improvement of Hall thruster performance. A typical one that should specifically be solved is the discharge current oscillation phenomenon observed under standard operational conditions at the high-voltage mode of the DC regime; it has been regarded as an unavoidable problem of Hall thrusters. Since the oscillation affects electron conductivity and anomalous diffusion in the acceleration channel, it appears to be a cause of reducing durability and of unstable operation. In particular, since long-time stable operation is required in a space mission, the development of a thruster design to probe the physical mechanisms of discharge current oscillation phenomenon is an urgent item. ${ }^{1,2,6-12)}$

The present research focuses on the large-amplitude and low-frequency discharge current oscillation in $20 \mathrm{kHz}$-range ( $20 \mathrm{kHz}$-range oscillation), which is speculated to be a main cause for the deterioration of propulsive efficiency and operational instability among various oscillation phenomena observed in Hall thrusters. To propose a technique to control the amplitude, the relationship between amplitude and ionization-zone length is analytically targeted by using an unsteady one-dimensional numerical analysis, where the optimization of operating conditions and the best device design would be provided after the oscillation phenomenon and plasma states in an acceleration channel had been clarified.

Since the Hall thruster was developed in Russia, as mentioned above, experimental and theoretical analyses have played major roles. In contrast, the numerical approaches performed in recent years are predicted to be important in future Hall thruster research. The numerical analyses especially related to oscillation phenomena have been performed by Martinez-Sanchez, ${ }^{6,8,13)}$ Boef, ${ }^{2,9,14)}$ and Arakawa, ${ }^{3,15)}$ et al. The development of numerical codes capable of simulating the characteristics of real thrusters enables us to properly understand the plasma parameters in the acceleration channel, where experimentally accurate measurements are usually difficult, and to discover a better thruster design and prompt evaluation of performance. In most papers, however, the calculated results give no quantitative or even qualitative agreements with experimental results because of imperfect models. Based on these situations, an attempt is given here to construct a numerical code containing the factors that have not been considered until now, which hopefully can help to explain the oscillation phenomenon as exactly as possible: (1) introducing diffusion terms into the electron momentum and energy equations; (2) including a time-derivative term in the electron energy equation; and (3) using a 3rd-orderaccurate scheme for space integration.

\section{Unsteady One-Dimensional Numerical Analysis}

\subsection{Numerical model}

Past works indicated that the $20 \mathrm{kHz}$-range oscillation was caused by a periodic ionization mechanism ${ }^{1,2,8)}$ in which the ionization process proceeded from anode toward the exit in an axial direction of the acceleration channel. Thus a simplified unsteady one-dimensional model for $20 \mathrm{kHz}$-range oscillation can be constructed. The concept of this analytical model is based on the acceleration part shown in Fig. 1,5) and a set of flow-field equations are composed of (1) mass conservation equations for ion and neutral, (2) a momentum equation for electron, (3) a momentum equation for ion, and (4) an energy balance equation for electron.

\subsection{Assumptions}

The following assumptions are made:

1) A radial magnetic field is nearly vertical in the axial direction of the acceleration channel.

2) The ion is generated by an electron-neutral inelastic collision, moving under electromagnetic force.

3) The ion is accelerated only by an axial electric field where a part of generated ions is lost by recombination with electrons on a channel wall.

4) An electron is emitted from the cathode, moving toward the anode by actions of the electric field and diffusions.

5) The electron distribution function is Maxwellian.

6) The plasma is quasi-neutral, where the electron number density is calculated from an ion mass conservation equation.

7) The neutral species flows at a constant velocity.

\subsection{Spatial distribution of magnetic field}

The axial distribution of the radial magnetic field used in the analysis is shown in Fig. 2. Plotted are the measured values (for different coil currents) at the radial middle of the

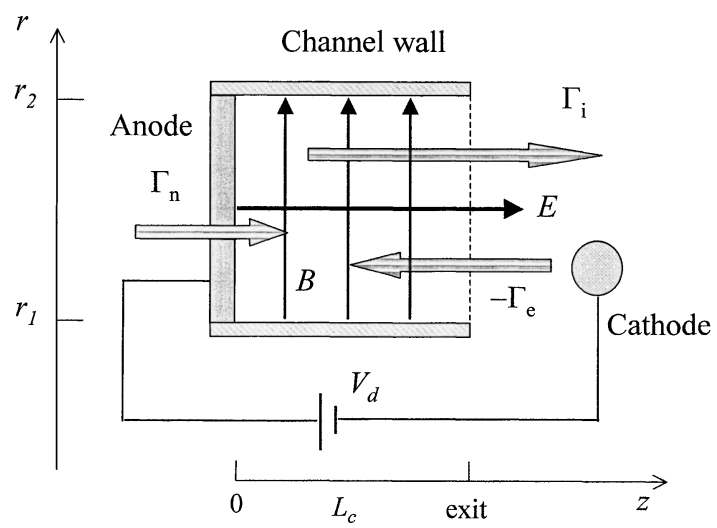

Fig. 1. A schematic of the acceleration part of a Hall thruster for one-dimensional numerical analysis: $\Gamma_{\mathrm{n}}, \Gamma_{\mathrm{i}}$, and $\Gamma_{\mathrm{e}}$ are axial fluxes of neutral, ion, and electron; $L_{\mathrm{e}}$ is the acceleration channel length, $r_{1}$ and $r_{2}$ are the inner and outer radii of the acceleration channel; $E$ is the axial electric field; $B$ is the radial magnetic field; $V_{\mathrm{d}}$ is discharge voltage. 


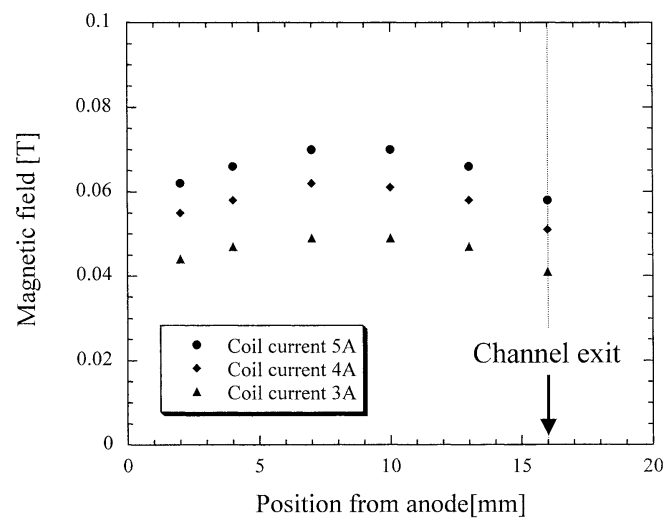

Fig. 2. The axial disrtribution of a radial magnetic field considered in calculation. The plots correspond to the values measured for different coil currents at the radial middle of the acceleration channel of the Nagoya University Hall thruster II.

acceleration channel of the Nagoya University Hall Thruster II, which is the so-called Japan-Type Hall Thruster6) ${ }^{6}$ originally designed by Komurasaki. ${ }^{3,16,17)}$ Its special aspect is that the magnetic field lines are distributed nearly flat during acceleration. The analytical expression for this magnetic field profile (coil current 4A) is approximated as

$$
B(z)=-\frac{1}{64} \times 10^{4} \times(z-0.008)^{2}+0.062 .
$$

\subsection{Fundamental equations}

\subsubsection{Conservation equations}

In an unsteady state, the conservation equations for ion and neutral species with production and loss terms of plasma given by Eqs. (2) and (3):

$$
\begin{gathered}
\frac{\partial n_{\mathrm{i}}}{\partial t}+\frac{\partial \Gamma_{\mathrm{i}}}{\partial z}=n_{\mathrm{e}}\left(v_{\mathrm{P}}-v_{\mathrm{L}}\right), \\
\frac{\partial n_{\mathrm{n}}}{\partial t}+\frac{\partial \Gamma_{\mathrm{n}}}{\partial z}=-n_{\mathrm{e}}\left(v_{\mathrm{P}}-v_{\mathrm{L}}\right) .
\end{gathered}
$$

Here, $\nu_{\mathrm{P}}=n_{\mathrm{n}}\left\langle\sigma v_{\mathrm{e}}\right\rangle_{\text {ion }}$, whereas $\left\langle\sigma v_{\mathrm{e}}\right\rangle_{\text {ion }}$ indicates the following ionization rate: ${ }^{18)}$

$$
\left\langle\sigma v_{\mathrm{e}}\right\rangle_{\text {ion }}=\sigma \sqrt{\frac{8 k T_{\mathrm{e}}}{\pi m_{\mathrm{e}}}}\left(1+\frac{e V_{\mathrm{i}}}{k T_{\mathrm{e}}}\right) \exp \left(-\frac{e V_{\mathrm{i}}}{k T_{\mathrm{e}}}\right),
$$

where,

$$
v_{L}=\left[2 /\left(r_{2}-r_{1}\right)\right] \sqrt{k T_{\mathrm{e}} / m_{\mathrm{i}}} \exp (-0.5) .^{5)}
$$

\subsubsection{Electron momentum equation}

The electron flow toward the anode across radial magnetic field lines is given by the momentum equation, taking into account the electric field and divergences of electron temperature and density:

$$
\Gamma_{\mathrm{e}}=-\mu_{\mathrm{e}} n_{\mathrm{e}} E-D_{\mathrm{e}} \frac{\partial n_{\mathrm{e}}}{\partial z}-D_{\mathrm{e}}^{\mathrm{T}} n_{\mathrm{e}} \frac{1}{T_{\mathrm{e}}} \frac{\partial T_{\mathrm{e}}}{\partial z} .
$$

Here the diffusion in the acceleration channel of the Hall thruster, where magnetic flux density is high (Tesla order), is much greater than the level expected from the classical diffusion theory, so that a Bohm-type diffusion coefficient that is a half-empirical formula is adopted as abnormal diffusion states: ${ }^{7)}$

$$
D_{\mathrm{e}}=\frac{k T_{\mathrm{e}}}{16 e B} .
$$

The thermal-diffusion coefficient is expressed by Eq. (8), using a density-diffusion coefficient:

$$
D_{\mathrm{e}}^{\mathrm{T}}=T_{\mathrm{e}} \frac{\partial D_{\mathrm{e}}}{\partial T_{\mathrm{e}}} .
$$

\subsubsection{Ion momentum equation}

The ion is not significantly affected by the magnetic field and is accelerated in a quasi-collisionless manner only by the axial electric field. The velocity of an ion generated by an ionization collision between electron and neutral is assumed to be equal to the neutral species velocity. Thus ion motion can be described by the following momentum equation:

$$
\frac{\partial \Gamma_{\mathrm{i}}}{\partial t}+\frac{\partial n_{\mathrm{i}} v_{\mathrm{i}}^{2}}{\partial z}=\frac{n_{\mathrm{i}} e E}{m_{\mathrm{i}}}+n_{\mathrm{e}}\left(v_{\mathrm{P}}-v_{\mathrm{L}}\right) v_{\mathrm{n}} .
$$

\subsubsection{Electron energy balance equation}

The energy balance equation for the electron can be written as ${ }^{5)}$

$$
\begin{aligned}
\frac{3}{2} \frac{\partial\left(k T_{\mathrm{e}} n_{\mathrm{e}}\right)}{\partial t}+\frac{5}{2} \frac{\partial\left(k T_{\mathrm{e}} \Gamma_{\mathrm{e}}\right)}{\partial z} \\
=-\zeta e \Gamma_{\mathrm{e}} E-(1+\beta) n_{\mathrm{e}} \nu_{\mathrm{P}} \varepsilon_{\mathrm{i}} \\
\quad-\left(\frac{2}{1-\delta}+\frac{1-2 \delta}{1-\delta} \chi\right) n_{\mathrm{e}} \nu_{\mathrm{L}} k T_{\mathrm{e}},
\end{aligned}
$$

where $\beta=2.0,{ }^{19)}$

$$
\delta\left(T_{\mathrm{e}}\right)=a\left(k T_{\mathrm{e}} / e\right)^{b} .
$$

( $a=0.141$ and $b=0.576$ for Boron Nitride and Xe) ${ }^{6)} \zeta$ is the ratio between the energy given to the thermal electron and the input power, which is equal to 1.0 if the electron distribution function is Maxwellian, and

$$
\chi=\frac{1}{4} \sqrt{8 m_{i} / \pi m_{\mathrm{e}}} \ln (1-\delta) \exp (0.5)
$$

\subsubsection{Numerical method}

These fundamental equations of the flow field are solved by a method suitable for this unsteady analysis as follows: The 3rd-order upwind scheme, which has a feature suppressing numerical oscillation and yet not necessitating too many lattice points, is used for the spatial integration of both ion and neutral species conservation equations. The energy balance equation for the electron is integrated by using the 4th order Runge-Kutta-Gill method for time integration where the accumulation of truncation error is suppressed.

\subsubsection{Calculation of electric field}

From the conservation equation for the ion Eq. (2) and the conservation equation for electron $i \rightarrow e$ in Eq. (2) under an electrically quasi-neutral assumption, the current conservation equation is obtained as follows:

$$
\frac{\partial\left(\Gamma_{\mathrm{i}}-\Gamma_{\mathrm{e}}\right)}{\partial z}=\frac{1}{e S} \frac{\partial I_{\mathrm{T}}}{\partial z}=0 .
$$

This equation indicates that the current depends only on time and not on space. Therefore the electric field for arbi- 
trary current is acquired from Eq. (6):

$$
E(z, t)=\frac{1}{\mu_{\mathrm{e}}(z, t) n_{\mathrm{e}}(z, t)}\left\{\frac{I_{\mathrm{T}}(t)}{e S}-n_{\mathrm{i}}(z, t) v_{\mathrm{i}}(z, t)\right\} .
$$

Thus the voltage is given by integrating the electric field as follows:

$$
\begin{gathered}
V_{\mathrm{e}}=-\int_{0}^{L_{\mathrm{c}}} E(z, t) d z, \\
V_{\mathrm{d}}=V_{\mathrm{c}}+V_{\text {exit }} .
\end{gathered}
$$

To determine the correct current for a given voltage $V_{\mathrm{d}}$, the current is altered and the calculations of Eqs. (14), (15), and (16) are repeated until the calculated voltage $\left(V_{\mathrm{c}}+V_{\text {exit }}\right)$ agrees with $V_{\mathrm{d}}$.

\subsubsection{Boundary conditions}

Since the results of calculation are strongly dependent on boundary conditions, boundary values as reasonable as possible are adopted. In other words, according to the experiment the ion density at anode is set at nonzero $5.0 \times$ $10^{16} \mathrm{~m}^{-3}$, and the electron temperature at channel exit is considered to be $3.5 \mathrm{eV}$ (a 10th of the voltage still remaining at the channel exit $[\fallingdotseq 35 \mathrm{~V}]$; this looks valid from the viewpoint that the electron temperature in the Hall thruster is nearly a tenth of the voltage $e^{5)}$ ). Note that the equilibrium discharge current after a quasi-steady state is reached is independent of initial conditions.

\section{Results and Discussion}

\subsection{Effect of diffusion terms}

In the present study, we introduce the diffusion terms (density-diffusion and thermal-diffusion) into the electron momentum and energy balance equations, which is the first attempt among these unsteady one-dimensional numerical analyses. First, with the introduction of the density-diffusion term, the amplitude of discharge current oscillation in the $20 \mathrm{kHz}$-range obtained by calculation (Fig. 3) closely approaches the one observed experimentally, as shown in

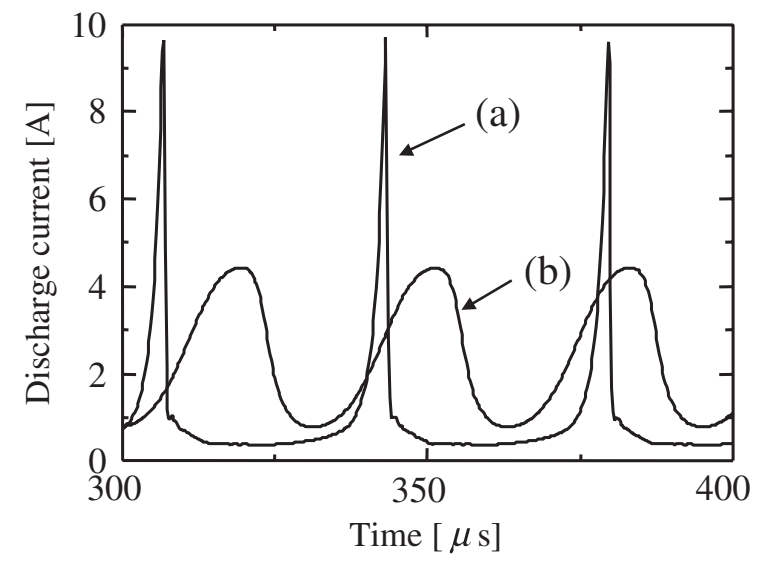

Fig. 3. The time evolution of discharge current predicted by calculation (a) without a diffusion term, and (b) with a diffusion term, for $V_{\mathrm{d}}=200 \mathrm{~V}$, $\dot{m}=1.38 \mathrm{mg} / \mathrm{s}, B=0.062 \mathrm{~T}, L_{\mathrm{c}}=16 \mathrm{~mm}$, and $T_{\mathrm{n}}=1,000 \mathrm{~K}$.

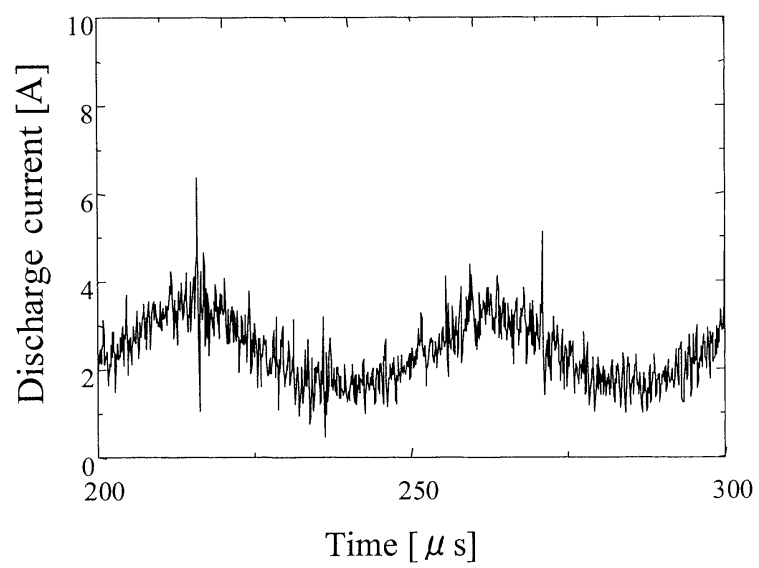

Fig. 4. The time evolution of discharge current obtained experimentally for the same conditions as in calculation (except for neutral species temperature).

Fig. 4. In other words, in comparison with the case of no diffusion term the amplitude decreases down to approximately half. The reason appears to be that the spatial dispersion of plasma is promoted by density-diffusion where the local ionization is relaxed, and as a result the rise of plasma density at the time of ionization is moderated. Second, even if the thermal-diffusion term is included, little difference is seen in amplitude. This result seems to be valid because the thermal-diffusion term is smaller than the other terms by orders of magnitude. Therefore it is concluded that the densitydiffusion besides the electric field plays an important role in the motion of electron in the acceleration channel; thus the inclusion of diffusion terms is necessary in the study of these and similar oscillation phenomena.

\subsection{Definition of ionization-zone length}

To discuss the relationship between amplitude and ionization-zone length in an unsteady one-dimensional numerical analysis, a new parameter "equilibrium length of ionization zone" is introduced.

Figure 5 shows the spatial distribution of neutral species density in the acceleration channel at a certain time (corresponding to point (2) in Fig. 10). Since ionization arises immediately behind the anode in our thruster, as shown in

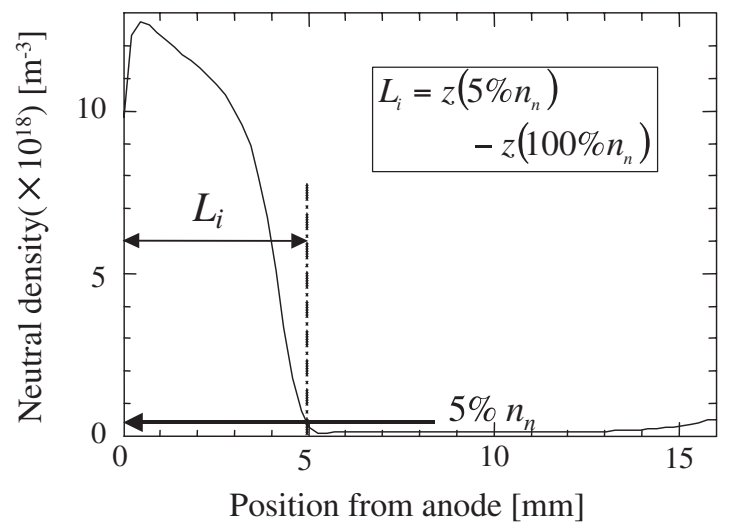

Fig. 5. The axial distribution of neutral species density in the acceleration channel at a certain time, where the definition of ionization-zone length is given. 


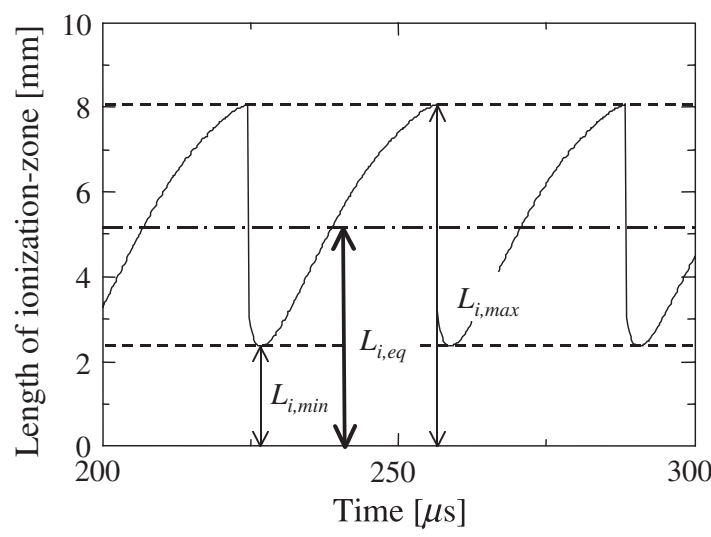

Fig. 6. The time evolution of ionization-zone length and the difinition of equilibrium length of ionization-zone.

Fig. 5, the position of the anode is considered to be the start position of ionization. Furthermore, the position where $95 \%$ of the initial neutral species supplied from the anode has been consumed by ionization is assumed to be the ionization completion position. In consequence, the ionization-zone length $L_{\mathrm{i}}$ is defined by the distance between the position of the anode and the position where neutral species density decreases to $5 \%$ of the neutral species density injected from the anode. The ionization-zone length then turns out to be a function of time, as shown in Fig. 6; thus the equilibrium

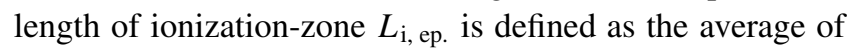
maximum and minimum ionization-zone lengths, as calculated by Eq. (17):

$$
L_{\mathrm{i}, \text { eq. }}=\left(L_{\mathrm{i}, \max }+L_{\mathrm{i}, \min }\right) / 2 .
$$

\subsection{Dependency on voltage}

Dependency of both frequency and amplitude of $20 \mathrm{kHz}-$ range oscillation on discharge voltage, which is the most important operating parameter of the Hall thruster, is studied. With regard to frequency, the calculated and experimental results both increase with discharge voltage after the onset of the $20 \mathrm{kHz}$-range oscillation regime, as shown in Fig. 7.

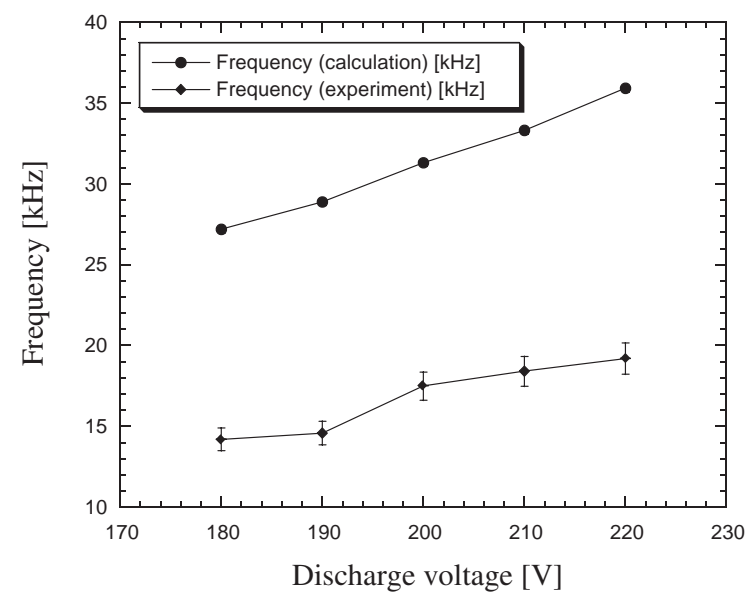

Fig. 7. A comparison between calculated and experimental frequencies as a function of discharge voltage for $\dot{m}=0.38 \mathrm{mg} / \mathrm{s}, B=0.062 \mathrm{~T}$, $L_{\mathrm{c}}=16 \mathrm{~mm}$, and $T_{\mathrm{n}}=1,000 \mathrm{~K}$ (only in calculation).

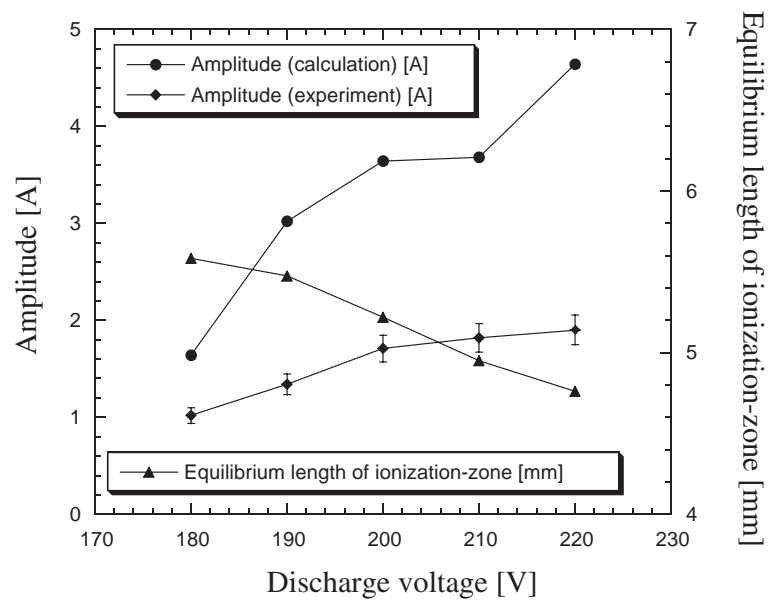

Fig. 8. The dependency of calculated and experimental amplitudes and equilibrium length of the ionization zone on discharge voltage for $\dot{m}=0.38 \mathrm{mg} / \mathrm{s}, B=0.062 \mathrm{~T}, L_{\mathrm{c}}=16 \mathrm{~mm}$, and $T_{\mathrm{n}}=1,000 \mathrm{~K}$ (only in calculation).

Figure 8 shows the dependency of (1) amplitudes of calculation and experiment and (2) equilibrium length of ionizationzone on discharge voltage; both amplitudes $\left(I_{\max }-I_{\min }\right)$ increase with discharge voltage. On the other hand, the equilibrium length of the ionization zone decreases as discharge voltage increases. The reason is that the ionization-zone length shrinks as discharge voltage increases, leading to a surge in the upper limit of plasma density at the time of rapid ionization, thus elevating the amplitude (refer to 1 ) for detailed reasons. So if this result is interpreted vice versa, the extension of ionization-zone length would cause a decrease in amplitude. Therefore a method controlling amplitude from the viewpoint of ionization-zone length may be newly proposed.

\subsection{Dependency on neutral temperature}

In Section 3.3, our calculation gave a qualitative agreement with experiments, but not a quantitative one. A main reason for disagreement can be (1) the wrong assignment of neutral species temperature, or (2) simply the experimental errors. In the present analysis, the neutral species temperature is set at $1,000 \mathrm{~K}$ without rigorous theoretical background; it is usually difficult to accurately measure the temperature of neutral species incoming to the ionization zone, and the actual temperature of neutral species would be higher than the standard value, since the neutral sonic-inlet in the ionization zone is heated by the anode, electron, channel wall, and others. ${ }^{1)}$ If the neutral species temperature is set higher, the frequency lowers and the amplitude gets smaller. As a result, the calculated results approach the experiments.

In this manner, although it is unavoidable to set the neutral species temperature as a boundary condition in calculation, the oscillation in the $20 \mathrm{kHz}$ range turns out to be sensitive to the temperature of neutral species incoming to the ionization zone. When the dependency of amplitude on neutral species temperature is calculated, it is interesting that the amplitude decreases with the neutral species temperature, as shown in Fig. 9. When this result is practically interpreted, the ampli- 


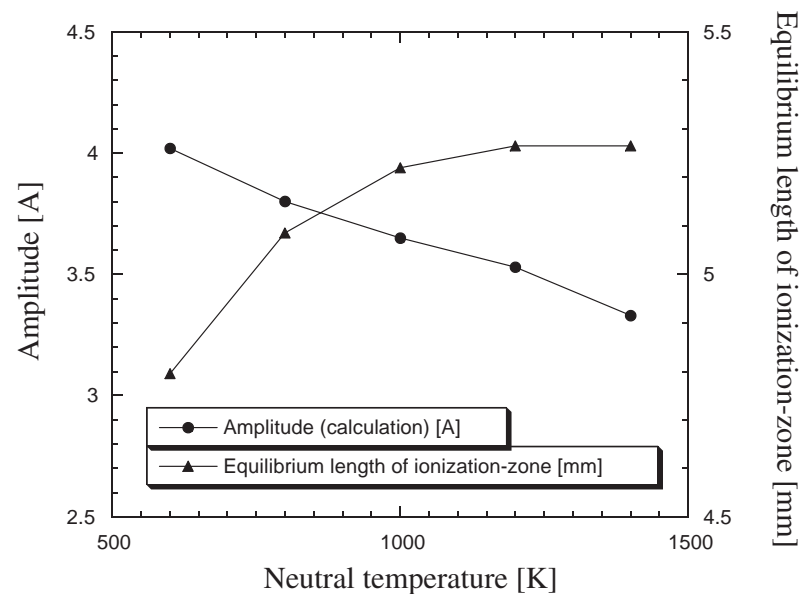

Fig. 9. The dependency of the calculated amplitude and equilibrium length of the ionization zone on neutral species temperature for $V_{\mathrm{d}}=200 \mathrm{~V}, \dot{m}=1.38 \mathrm{mg} / \mathrm{s}, B=0.062 \mathrm{~T}$ and $L_{\mathrm{c}}=16 \mathrm{~mm}$.
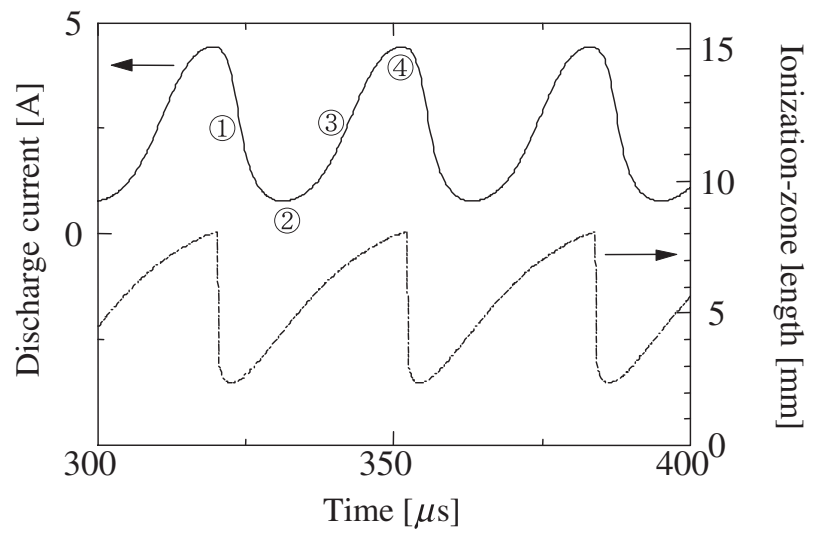

Fig. 10. The time evolution of discharge current and ionization-zone length for $V_{\mathrm{d}}=200 \mathrm{~V}, \dot{m}=1.38 \mathrm{mg} / \mathrm{s}, B=0.062 \mathrm{~T}, L_{\mathrm{c}}=16 \mathrm{~mm}$, and $T_{\mathrm{n}}=1,000 \mathrm{~K}$.

tude may be decreased if the neutral species temperature incoming to the ionization zone is raised. Namely, because the sound speed of the neutral species increases with the neutral temperature, it moves the ionizing completion position toward the direction of the channel exit; thus the ionization zone length increases. Consequently the rapid rise of plasma density at the time of ionization is suppressed and the amplitude decreases. The neutral species temperature can therefore be changed as a method of controlling the amplitude of $20 \mathrm{kHz}$ range oscillation.

\subsection{Thruster design in terms of ionization-zone length}

In this section, the time evolution of plasma and the electromagnetic field in the periodic ionization process that seems to be a cause of $20 \mathrm{kHz}$ range oscillation is studied, and the theoretically best design of the Hall thruster is discussed, especially from the viewpoint of the ionization-zone length.

Figure 10 shows the time evolution of discharge current and ionization-zone length. Figures 12.1-12.4 are the spatial distribution of electron temperature and plasma density in the acceleration channel at the feature points (1)-(4) of discharge current oscillation, shown in Fig. 10. The spatial dis-

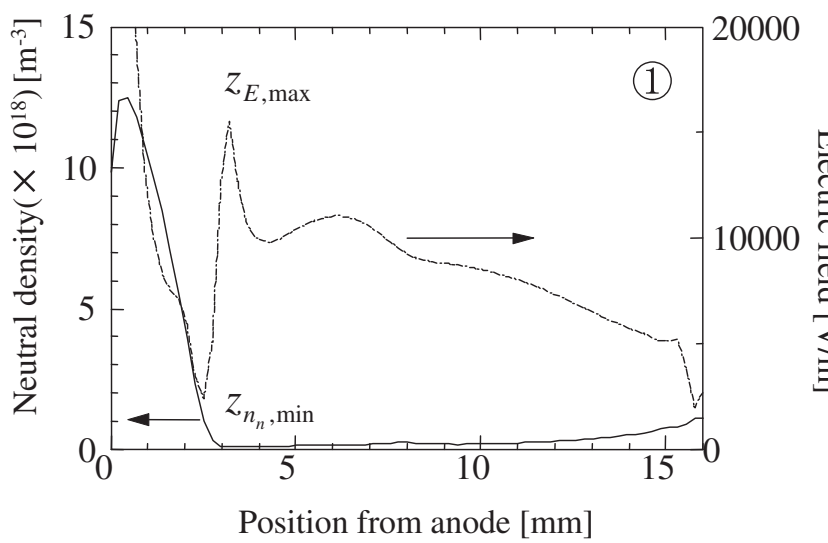

Fig. 11. The spatial distribution of neutral species density and electric field at point (1) in Fig. 10.
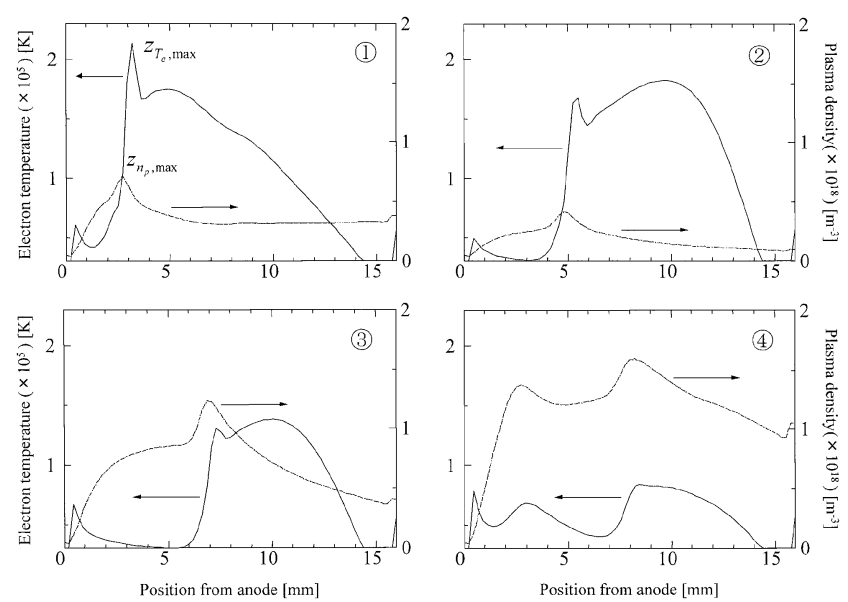

Fig. 12. The spatial distribution of the electron temperature and plasma density in the acceleration channel at points (1)-(4) of discharge current oscillation, shown in Fig. 10.

tribution of neutral species density and electric field at point (1) in Fig. 10 is represented in Fig. 11.

First of all, as seen in Figs. 12.1 and 11, the position that neutral species density comes down to $5 \%$ of the initial neutral species density injected from anode $z_{\mathrm{n}_{\mathrm{n}}}$,min, which corresponds to the ionization completion position as defined in Section 3.2, is nearly in agreement with both the position of maximum plasma density $z_{\mathrm{n}_{\mathrm{p}} \text {, max }}$ and the position of maximum electron temperature $z_{\mathrm{T}_{\mathrm{e}} \text {, max }}$ : The turning point where the increasing tendency changes to the decreasing one, viewing from the channel exit, which is caused by consuming for ionization the energy that the electrons moving toward the anode have acquired from the electric field. This relationship $\left(z_{\mathrm{n}_{\mathrm{n}}, \min } \fallingdotseq z_{\mathrm{n}_{\mathrm{p}}, \max } \fallingdotseq z_{\mathrm{T}_{\mathrm{e}}, \max }\right)$ is recognized for points (2)-4), as shown in Figs. 12.2-12.4 (although the relevant figures are not shown in this paper). Therefore, because of the prediction that the amplitude decreases by extending the ionization-zone length, it is preferable to suppress the amplitude to place the position $z_{\mathrm{n}_{\mathrm{p}} \text {, } \max } \fallingdotseq z_{\mathrm{T}_{\mathrm{e}} \text {, } \text { max }}$ closest to an exit side.

Thus an idea satisfying this requisite in thruster design can be examined theoretically. Again from Figs. 12.1 and 11, 
it is seen that the position $z_{\mathrm{n}_{\mathrm{p}} \text {, max }} \fallingdotseq z_{\mathrm{T}_{\mathrm{e}} \text {, max }}$ nearly agrees with the position of maximum electric field $z_{\mathrm{E} \text {,max }}$. Moreover, this position $z_{\mathrm{E}, \max }$ is nearly equal to the position of maximum magnetic field $z_{\mathrm{B} \text {, max }}$. Namely, the electric field reaching maximum at position $z_{\mathrm{B} \text {, max }}$ enhances the electron diffusion because of current continuity, minimizing the electron restriction by the magnetic field. Therefore in the acceleration channel of constant length (long enough to complete ionization in terms of propellant efficiency), placing the maximum position of coil volumes number $z_{\mathrm{c}_{\mathrm{n}} \text {, max }}$ closest to an exit side, which corresponds to the maximum position of magnetic field $z_{\mathrm{B} \text {, max }}$, leads to an extension of ionizationzone length and as a result causes a decrease of amplitude. In this manner, placing the maximum positions of coil volumes number $z_{\mathrm{c}_{\mathrm{n}} \text {, max }}$ closest to an exit side helps to decrease the loss of plasma on the wall behind the ionizing position and to increase the amount of ion beam extraction, which is desirable from the viewpoint of acceleration efficiency.

In summary, according to the qualitative discussions mentioned above for a better thruster design it is desirable that the position of maximum magnetic field $z_{\mathrm{B} \text {, max }}$ should be placed closest to an exit side from the viewpoint of controlling the amplitude and raising the acceleration efficiency; it is necessary that the acceleration channel be of minimum length, just enough to complete ionization in view of propellant efficiency.

\section{Conclusion}

As a step to ward the main goal of proposing a method to control the amplitude of $20 \mathrm{kHz}$ range oscillation, an unsteady one-dimensional numerical analysis was performed in the axial direction of the acceleration channel, which resulted in these findings:

- The amplitude of $20 \mathrm{kHz}$ range oscillation can be controlled by changing the ionization-zone length.

- Through the introduction of the density-diffusion term into electron momentum and electron energy balance equations, the calculated oscillation behaviors approached the one observed experimentally.

- A possibility was suggested that raising the temperature of neutral species incoming to the ionization zone will enable us to decrease the amplitude.

- A numerical code estimating more exactly the oscillation phenomenon and physical quantities in plasma has been developed; its usefulness was verified through a comparison with experimental results.

- The time evolution of plasma and the electromagnetic field during a periodic ionization process was examined, and it was learned that the position of maximum magnetic field should be placed closest to an exit side.

The studies based on the present numerical code of which the effectiveness is verified here are soon planned. For ex- ample, (1) the dependency of amplitude on magnetic field strength, distribution of magnetic field, and mass flow rate of propellant; (2) estimation of performance (acceleration efficiency, energy efficiency, and propellant efficiency); and (3) development of an analytical model that makes it possible to capture more practically the plasma phenomena in a narrow acceleration part, where experimental examination is limited because of the difficulties in detailed measurements.

\section{Acknowledgement}

The authors would like to acknowledge Mr. Akira Saito for his assistance in the experiments.

\section{References}

1) Furukawa, T., Miyasaka, T., Sakurai, Y. and Fujiwara, T.: Measurement and Modeling of Low Frequency Oscillation in a Hall Thruster, ISTS-00-b-22 (2000).

2) Boeuf, J. P. and Garritues, L.: Low Frequency Oscillation in a Stationary Plasma Thruster, J. Appl. Phys., 84 (1998), pp. 3541-3554.

3) Komurasaki, K. and Arakawa, Y.: Two-Dimensional Numerical Model of Plasma Flow in a Hall Thruster, J. Propul. Power, 11 (1995), pp. 1317-1323.

4) Jankovsky, R., Tverdokhlebov, S. and Manzella, D.: High Power Hall Thrusters, AIAA-99-315745 (1999).

5) Hirakawa, M.: Plasma Particle Simulation in Electric Propulsion, Tokyo-Univ., Ph.D. Thesis, 1995.

6) Fife, J. M., Martinez-Sanchez, M. and Szabo, J.: A Numerical Study of Low-Frequency Discharge Oscillations in Hall Thrusters, AIAA97-3052 (1997).

7) Hirakawa, M.: Electron Transport Mechanism in a Hall Thruster, IEPC-97-021 (1997).

8) Martinez-Sanchez, M., Ahedo, E. and Noguchi, R.: Linear 1-D Analysis of Oscillations in Hall Thrusters, IEPC-99-105 (1999).

9) Garritues, L. and Boeuf, J. P.: Characteristics of the SPT100-ML Comparisons between Experiments and Models, IEPC-99-102 (1999).

10) Baranov, V. I., Nazarencko, Yu. S., Petrosov, V. A., Vasin, A. I. and Yashnov, Yu. M.: Theory of Oscillations and Conductivity for Hall Thruster, AIAA-96-3192 (1996).

11) Hruby, V., Connolly, W., Monheiser, J., Pote, B. and Freeman, C.: DC-DC Converter for Hall Thruster Plasma Discharge, IEPC-99-061 (1999).

12) Choueiri, E. Y.: Characterization of Oscillations in Closed Drift Thrusters, AIAA-94-3013 (1994).

13) Fife, J. M. and Martinez-Sanchez, M.: Two-Dimensional Hybrid Particle-In-Cell (PIC) Modeling of Hall Thrusters, IEPC-95-033 (1995).

14) Garritues, L., Boyd, I. D. and Boeuf, J. P.: Computation of Hall Thruster Performance, IEPC-99-098 (1999).

15) Arakawa, Y., Komurasaki, K. and Hirakawa, M.: Hall Thruster, Aeronautical and Space Science Japan, 46 (1998), pp. 146-152.

16) Komurasaki, K.: Propulsive Performance and Acceleration Process of Hall Thrusters, Tokyo-Univ., Ph.D. Thesis, 1992.

17) Mikami, K., Komurasaki, K. and Fujiwara, T.: Optimization of Channel Configuration of Hall Thrusters, IEPC-95-033 (1995).

18) Mikami, K. and Fujiwara, T.: Experiment for Optimization of Channel Configuration in Hall Type Thrusters, Nagoya-Univ., M. Thesis, 1995.

19) Smirnov, V. A.: Electron Energy Balance in a Hall-Current Accelerator with Extended Acceleration Zone, Sov. J. Plasma Phys., 5 (1979), pp. 202-205. 